\title{
CARMEN : une plateforme de caractérisation métrologique dédiée aux nanomatériaux
}

\section{CARMEN : a metrological characterization platform for nanomaterials}

\author{
Nicolas FELTIN, Alexandra DELVALLÉE, Sébastien DUCOURTIEUX, Pierre GOURNAY, Benoît POYET, \\ Myriam TRABELSI, Younes BOUKELLAL et François PIQUEMAL
}

Laboratoire national de métrologie et d'essais (LNE), 29 Avenue Roger Hennequin, 78197 Trappes Cedex, France, nicolas.feltin@1ne.fr.

\begin{abstract}
Résumé
Le LNE développe une plate forme de CARactérisation MEtrologique des Nanomatériaux (CARMEN) afin de proposer une offre complète de mesure des principaux paramètres caractérisant un nanoobjet (taille, forme, polydispersité, composition chimique, état d'agrégation/agglomération, charge en surface, surface spécifique, etc.). D'autres propriétés physiques telles que des mesures électriques locales pourront être effectuées sur des nanodispositifs. L'objectif de cette plateforme consiste à établir les différentes chaînes de traçabilité, à définir des protocoles de mesure et des méthodes d'échantillonnage afin d'être capable d'associer à chaque mesure une incertitude qui permettra d'offrir aux utilisateurs de la plateforme un niveau de confiance optimum. L'instrumentation est installée en salle blanche et en environnement contrôlé (température, humidité, vibration, etc.). Avec cette plateforme, le LNE sera en capacité d'aider les industriels et les laboratoires à répondre à la demande gouvernementale de déclaration des substances à l'état nanoparticulaire conformément au décret n ${ }^{\circ} 2012-232$ du 17 février 2012 (JORF n ${ }^{\circ} 0043$ du 19 février 2012). La plateforme permettra en outre de soutenir les équipes impliquées dans l'évaluation des risques liés aux nanomatériaux.
\end{abstract}

MOTS CLÉS : NANOTECHNOLOGIE, MÉTROLOGIE, ÉTUDE TOXICOLOGIQUE, ÉTUDE ÉCO-TOXICOLOGIQUE, PLATEFORME DE NANOCARACTÉRISATION, AFM, MAFM, MEB.

\footnotetext{
Abstract

LNE is developing a MEtrological ChARacterization of Nanomaterials (CARMEN) platform to offer a complete supply of main parameter measurements characterizing a nano-object (size, shape, polydispersity, agglomeration/aggregation state, surface charges, specific charges...). Other physical properties such local electrical measurements will be able to be performed on nanodevices. This platform aims to establish tracability routes, define measurement protocols and sampling methods. The uncertainties associated with measurements will offer to users an
}

optimum confidence level. Instrumentation is put in a clean room within a controlled environment (temperature, humidity, vibration...). Thanks to this platform, LNE will be able to help manufacturers and laboratories to meet to the governmental requests concerning the mandatory declaration of nanosubstances as described in the French decree number 2012-232, dated the 17th on February, 2012 (JORF number 0043 on 19th February 2012). Furthermore, this platform will make it possible to support the teams involved in the toxicological investigations linked to nanomaterials.

KEY WORDS: NANOTECHNOLOGY, METROLOGY, TOXICOLOGY, ECO-TOXICOLOGY, NANOCARACTERIZATION PLATFORM, AFM, MAFM, SEM.

\section{Introduction}

L'engouement actuel autour des nanomatériaux et le vaste champ d'applications que l'on peut en attendre s'expliquent par la forte dépendance des propriétés physico-chimiques des nano-objets avec leurs dimensions et leur morphologie. Ainsi, pour chaque propriété fonctionnelle souhaitée (électronique, magnétique, optique, thermodynamique, mécanique...) associée à une application particulière (tous les secteurs d'activités sont concernés), il est possible de concevoir «à la carte » un nano-objet qui lui correspond.

L'ensemble des acteurs impliqués dans ce domaine émergent s'accorde sur le fait que le développement d'une métrologie et d'une instrumentation adaptée au domaine nanométrique $(1 \mathrm{~nm}-100 \mathrm{~nm})$ aurait un effet «catalyseur» sur le développement global des nanotechnologies [co-nanomet]. 
L'instrumentation et les techniques de mesure à l'échelle du nanomètre jouent tout d'abord un rôle crucial dans le domaine de la recherche fondamentale en permettant d'accroitre les connaissances sur les propriétés intimes des nanomatériaux. Les chercheurs ont aussi besoin d'instruments pour corréler les paramètres physicochimiques de ces nano-objets avec les propriétés physiques nouvelles qui apparaissent à ces échelles afin d'en déterminer les paramètres limites. Mais surtout la nanométrologie suscite un intérêt croissant dans le monde industriel qui attend des outils précis pour un meilleur contrôle des processus de fabrication et une amélioration des systèmes qualités. Les micro- et nanotechnologies font actuellement face à une demande croissante de mesures quantitatives afin de conforter la fiabilité, la compétitivité et même la sécurité des produits manufacturés et des services.

Dans le même temps, de nombreux produits industriels tirant partie des propriétés uniques des nanomatériaux sont déjà sur le marché, et leur nombre ne cesse de croître. La production et l'utilisation croissante de nano-objets conduisent inévitablement à leur accumulation dans l'environnement. Ces nano-objets sont susceptibles de générer des effets néfastes sur la santé humaine ou l'environnement. En attendant l'élaboration de normes spécifiques, de nombreux rapports émanant d'agences gouvernementales [1-5] soulignent le manque d'outils ou de méthodes adaptés à la mesure des nano-objets, freinant ainsi les études sur l'évaluation des risques liés à ces nouveaux matériaux. Plus encore, les agences de surveillance ou de régulation des produits de consommation insistent sur l'absence de mesures fiables et reproductibles à l'origine de résultats parfois contradictoires. Les besoins couvrent l'instrumentation, les matériaux de référence, les protocoles, l'étalonnage et la formation.

Dans ce contexte, le LNE développe depuis 2010 une plateforme de CARactérisation MEtrologique des Nanomatériaux (CARMEN) qui permettra à terme de mettre en place des chaînes de traçabilité adaptées à la caractérisation des nano-objets et des nanosystèmes. Cette plateforme, unique en France, installée dans un environnement contrôlé, sera mise au service de l'industrie et du monde académique. Elle a pour objectif principal de développer une métrologie primaire des nanomatériaux. Elle permettra aussi de soutenir les études toxicologiques et éco-toxicologiques en cours sur l'évaluation des risques liés à l'utilisation des nanoproduits. À terme, elle sera constituée d'un ensemble d'instruments (AFM, MEB, DRX, BET, potentiométrie zéta, etc.) nécessaire pour la mesure des principaux paramètres physico-chimiques ainsi que d'instruments adaptés aux mesures locales électriques de nanosystèmes développés en micro- et nanoélectronique.

La mesure à l'échelle du nanomètre est ainsi devenue un défi à relever par les instituts nationaux de métrologie (INM) et le développement des nanosciences et nanotechnologies va immanquablement engendrer dans les années à venir une très grande activité métrologique qui concernera toutes les grandeurs physiques et chimiques.

\section{Nanomatériaux : définition et terminologie}

À ce jour, il n'existe pas de définition figée des nanomatériaux qui soit reconnue au niveau international. De nombreuses définitions ont été proposées par divers organismes nationaux ou internationaux mais celles-ci varient en fonction des domaines d'activités ou des enjeux économiques ou scientifiques sous-jacents.

L'organisation internationale de normalisation (ISO), dans le cadre des travaux effectués par son comité technique en charge de la normalisation des nanomatériaux (TC229), a défini un nanomatériau uniquement par une taille affectée à une dimension externe (nano-objet) ou interne (matériau nanostructuré) [6] :

- un nano-objet possède une ou plusieurs dimensions caractéristiques externes dans le domaine du nanomètre ;

- un matériau nanostructuré n'a aucune dimension externe dans le domaine nanométrique mais il est composé de parties nanométriques ou il est constitué en surface de motifs nanométriques.

En accord avec son homologue européen (CEN/ TC 352, European Standardisation Committee), un cadre général de taille a été proposé pour définir le domaine nanométrique compris approximativement entre $1 \mathrm{~nm}$ et $100 \mathrm{~nm}$. Une limite inférieure a été fixée afin d'exclure du domaine des nanomatériaux, les molécules et les atomes.

Cependant, de nombreux organismes à travers le monde critiquent l'idée d'imposer une limite supérieure au domaine (environ $100 \mathrm{~nm}$ ) car cela empêcherait de prendre suffisamment en compte des questions de distribution en taille et surtout certaines propriétés spécifiques (électriques, mécaniques, optiques, magnétiques, etc.) rencontrées à l'échelle submicrométrique. En effet, la révolution annoncée des nanomatériaux réside dans la grande dépendance qui existe entre leurs propriétés physico-chimiques et leurs caractéristiques géométriques (taille et forme). De plus, pour une composition chimique et une structure cristallographique données, ces propriétés peuvent être très différentes de celles du matériau à l'état massif : des propriétés quantiques peuvent par exemple intervenir en dessous de $20 \mathrm{~nm}$ dans les semiconducteurs. Par conséquent, certains organismes souhaiteraient que cette modification des propriétés du matériau soit pleinement incluse dans la définition des nanomatériaux. Ce critère est parfois simplement ajouté au critère de taille et dans d'autres cas est largement mis en avant. Il existe une définition non-officielle provenant de rapports américains qui stipule que le domaine nanométrique se situe entre «l'état moléculaire et l'état massif, ce qui est généralement, mais pas exclusivement, le cas en dessous de $100 \mathrm{~nm}$ et au-dessus de $1 \mathrm{~nm}$... ». Cette formulation permet de ne pas exclure du domaine des matériaux qui présenteraient des propriétés originales mais qui possèderaient des dimensions caractéristiques supérieures à $100 \mathrm{~nm}$. 
L'ACC (American Chemistry Council) a une approche inverse du problème en proposant d'exclure du domaine des nanomatériaux, «les matériaux qui ne présentent aucune propriété originale/nouvelle/unique comparée à la forme massive du matériau de même composition chimique » [7].

Enfin, la Communauté Européenne a fait paraître une recommandation datée du 6 octobre 2011 concernant la définition des nanomatériaux, qui stipule qu'une substance est définie comme «nanomatériau» si elle est constituée de $50 \%$ de particules dont la taille est comprise entre $1 \mathrm{~nm}$ et $100 \mathrm{~nm}$ [8].

\section{La mesure : un catalyseur pour le développement des nanotechnologies}

L'idée communément répandue dans le domaine des nanosciences et nanotechnologies est que la rupture technologique à laquelle les industriels doivent faire face se situe au niveau de la fabrication. En réalité, dans de nombreux secteurs, élaborer des dispositifs et des matériaux de plus en plus petits est le fruit d'un long processus continu. C'est ce que décrit un rapport de la Royal Society $\mathcal{E}$ Royal Academy of Engineering qui conclut que la véritable rupture technologique pour le monde industriel se situe plutôt «au niveau des outils utilisés pour observer et mesurer les propriétés, et contrôler la fabrication à l'échelle nanométrique » [9].

\subsection{Les besoins dans le secteur industriel}

Dans le domaine des nanotechnologies, le secteur industriel doit être en capacité de mesurer à l'échelle du nanomètre, la taille, la forme et les principales propriétés physiques et chimiques afin de produire des nanomatériaux et des nanodispositifs avec un haut degré de précision et une grande fiabilité. Ceci impose qu'aux niveaux national et international une chaîne de traçabilité et d'étalonnage spécifique soit mise en place pour chacune des grandeurs considérées. Or la nanométrologie, c'est-à-dire la science de la mesure à l'échelle du nanomètre, n'en est qu'à ses balbutiements. Même si les instituts nationaux de métrologie européens possèdent des instruments de mesure, les méthodes sont seulement en cours de développement et les matériaux de références font cruellement défaut. Cela rend souvent impossible la mise en place des tests d'inter-comparaisons pour échanger ou comparer des données de mesures. Le manque d'étalons de transfert et de matériaux de référence avait été souligné dès $2002[10,11]$. De plus, peu de normes sont actuellement disponibles concernant la mesure des paramètres physico-chimiques et un grand chantier s'ouvre dans ce domaine.

L'industrie du semi-conducteur est un secteur industriel clé de nos sociétés modernes qui a su évoluer dans son approche métrologique et mettre en place des moyens de mesure sur les lignes de production. La principale motivation de ce secteur repose sur une augmentation permanente de la vitesse de calcul des microprocesseurs et de la capacité d'enregistrement des dispositifs. Depuis l'avènement de la microélectronique, le respect de la loi de Moore impose de diminuer la taille des transistors en réduisant l'épaisseur des grilles et des oxydes de grilles. En 1971, le microprocesseur Intel's 4004 était constitué de 2300 transistors; en 2003, 108 milliards de transistors sont intégrés dans l'Intel Xenon. Les recherches actuelles se concentrent sur des technologies dont les dimensions critiques (CD, critical dimension) sont inférieures à $32 \mathrm{~nm}$ (gravure à $22 \mathrm{~nm}$ pour la génération de processeurs Ivy Bridge d'Intel ou à $28 \mathrm{~nm}$ pour le processeur graphique Kepler de Nvidia, les prévisions pour 2013 sont proches de $14 \mathrm{~nm}$ ) [12]; le CD représente la taille de la structure la plus petite que le process permet de fabriquer. Il s'agit d'un paramètre déterminant pour l'industrie du semi-conducteur. Or, les alignements d'objets, les épaisseurs, les tolérances en termes de CD et les incertitudes de mesures recherchées sont déjà de l'ordre du nanomètre. Ainsi, depuis peu, afin de maîtriser la fabrication d'objets de plus en plus petits, des stratégies métrologiques se mettent en place et la nécessité de raccorder ces mesures à la définition du mètre devient primordiale. C'est la raison pour laquelle, les documents de l'ITRS (International Technology Roadmap for Semiconductors), la très exigeante feuille de route des technologies de l'information et de la communication (TIC), intègrent pleinement des stratégies complexes de métrologie à l'échelle du nanomètre [13].

\subsection{Les besoins en santé et environnement}

De nombreux produits de consommation courante contenant des nanomatériaux sont déjà commercialisés (industrie automobile, produits cosmétiques, produits agro-alimentaires, jeux pour les enfants, chimie, électronique, santé, etc.), ce qui provoque l'inquiétude des pouvoirs publics pour la santé du consommateur et pour l'environnement. Les INM doivent contribuer à apporter des réponses aux questions légitimes que se posent les citoyens sur les risques potentiels associés aux nanoparticules.

Certaines études montrent que les nanomatériaux et notamment les nanoparticules présentent une toxicité bien différente de celle du matériau massif de même nature. Sur la vingtaine de milliards de dollars investis chaque année par les industriels dans les nanotechnologies, une part encore marginale est consacrée à l'estimation des risques environnementaux et sanitaires. D'après les deux rapports produits par l'AFSSET (Agence Française de Sécurité Sanitaire de l'Environnement et du Travail, devenue Agence nationale de sécurité sanitaire de l'alimentation, de l'environnement et du travail, Anses), certains dangers sont d'ores et déjà identifiés pour une exposition par voie cutanée, par ingestion ou plus fréquemment par inhalation [1,2]. En effet, la nanoparticule, de par ses propriétés dimensionnelles, peut franchir différentes barrières biologiques et pénétrer dans les systèmes vitaux.

Or, les procédures de régulation et de contrôle des substances mises sur le marché (notamment REACH) 
ne sont pas adaptées aux nanomatériaux car elles datent d'une époque où seule la composition chimique était prise en compte pour expliquer la toxicité des composés. Les agences de surveillance ou de régulation des produits de consommation insistent sur le manque de métrologie dans ce domaine et précisent que les «notions de dimensions et de morphologie seront déterminantes pour évaluer la toxicité des nanoparticules » [1-3].

Une démarche «nanotoxicologique» ambitieuse est nécessaire car les résultats des études toxicologiques et éco-toxicologiques sont peu nombreux, assez disparates et parfois contradictoires traduisant les lacunes du système actuel en termes de métrologie et de moyens de mesure. Les différents rapports des agences françaises et européennes de surveillance et régulation des produits de consommation (Anses, EFSA...) [1,3] préconisent un recensement précis et exhaustif des nanomatériaux existants et produits par les industriels, ainsi que la mise en place de structures d'enregistrement et de surveillance. Ces agences souhaitent obliger les entreprises à fournir des informations claires sur les nanoproduits qu'elles fabriquent. Dans une résolution concernant les aspects réglementaires des nanomatériaux, le Parlement Européen demande « urgemment la mise au point de protocoles d'essais et de normes en matière de métrologie adéquats permettant d'évaluer, sur la base d'une approche pluridisciplinaire, l'exposition des travailleurs, des consommateurs et de l'environnement aux nanomatériaux et les risques liés à ces derniers » [14].

Afin de favoriser l'émergence rapide de normes, le comité technique de l'ISO en charge de la normalisation des nanomatériaux (ISO/TC 229) a défini un ensemble de paramètres physico-chimiques déterminants pour l'étude des nano-objets fabriqués par l'homme et soumis aux tests toxicologiques. Cet ensemble de dix paramètres comprend :

- la taille;

- la distribution en taille;

- la forme;

- l'état d'agrégation ou d'agglomération;

- la composition chimique ;

- la structure cristallographique;

- la surface spécifique;

- la charge en surface;

- la chimie de surface;

- la solubilité/dispersibilité.

\subsection{Un décret relatif à la déclaration annuelle des substances à l'état nanoparticulaire}

Dans le cadre de loi dite «Grenelle 2 », le décret relatif à la prévention des risques pour la santé et l'environnement résultant de l'exposition aux substances à l'état nanoparticulaire, a été publié au Journal Officiel du 19 février 2012.
Ce décret impose que chaque fabricant, importateur et distributeur effectue une déclaration dès lors qu'il produit, importe ou distribue au moins $100 \mathrm{~g}$ par an d'une substance à l'état nanoparticulaire, en l'état ou contenue dans un mélange sans y être liée, ou de matériaux destinés à rejeter cette substance dans des conditions normales ou raisonnablement prévisibles d'utilisation. Cette déclaration doit être adressée chaque année, avant le $1^{\mathrm{er}}$ mai, au Ministre chargé de l'Environnement.

En complément à cette déclaration, un arrêté est paru au journal officiel daté du 10 août 2012, pris en application des articles R. 523-12 et R. 523-13 du code de l'environnement. Le fabricant, l'importateur ou le distributeur se doit de mesurer un ensemble de paramètres caractérisant la «nanosubstance » (taille des nanoparticules, distribution en taille, état d'agrégation/agglomération et forme). Cette mesure devra être accompagnée d'une incertitude et d'une description du protocole de mesure. L'état cristallin, la surface spécifique et la charge en surface doivent aussi être fournies si elles sont disponibles par le déclarant. L'ensemble des informations sera envoyé à l'ANSES qui a été mandaté par le gouvernement pour mettre en place une base de données. La liste des paramètres retenus pour cet arrêté s'inspire fortement des travaux de l'ISO/TC 229 relatif à la définition des dix paramètres (voir partie précédente). Pour le moment, seules la solubilité et la dispersibilité n'ont pas été retenues par les pouvoirs publics.

\section{Projet CARMEN du LNE : une plateforme dédiée à la caractérisation métrologique des nanomatériaux}

Pour répondre aux exigences industrielles en termes de nanométrologie mais aussi pour soutenir les études des nanomatériaux soumis aux tests toxicologiques, le LNE développe depuis deux ans une plateforme regroupant l'instrumentation nécessaire à la mesure des paramètres définis par l'ISO/TC 229 (voir §.3.2). L'objectif est de définir des protocoles de mesure validés et d'assurer la traçabilité aux unités SI (Système international d'unités) pour toutes les grandeurs concernées. Le manque de matériaux de référence est actuellement comblé par des collaborations avec des chimistes ou des physiciens qui aident les équipes du LNE à obtenir des nanoparticules ou des nanostructures de référence.

Le développement d'une métrologie primaire consacrée aux principaux paramètres définissant un nano-objet sera un travail de longue haleine mais la plupart des rapports indiquent que la nanométrologie dimensionnelle est une première étape déterminante : elle permet une mesure absolue et traçable des paramètres géométriques qui caractérisent un nano-objet. Actuellement, le travail de métrologie primaire se concentre donc en priorité sur le cœur dimensionnel de la plateforme. Il est constitué d'un AFM (Atomic Force Microscope) dit «métrologique » (mAFM), qui est l'instrument de référence national et de deux microscopes : microscope à force atomique et un microscope électronique à balayage (MEB ou SEM pour Scanning Electron Microscope). Dans les 


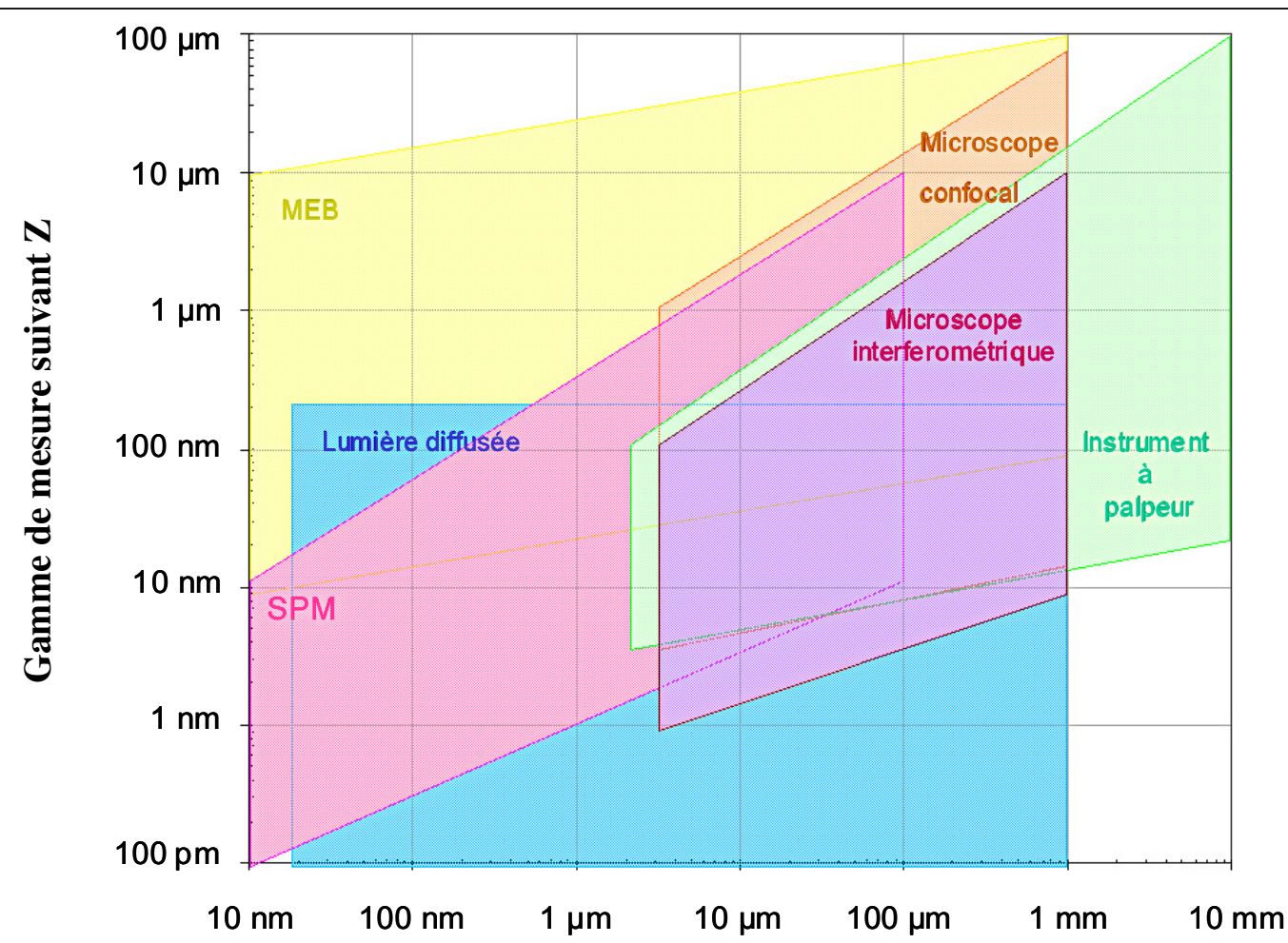

Gamme de mesure suivant XY

Fig. 1. - Gamme de mesure pour différentes familles d'instruments couramment utilisés en nanométrologie dimensionnelle.

parties suivantes, nous décrivons l'état de l'art de la mesure dimensionnelle à l'échelle du nanomètre, la chaîne de traçabilité associée à ces mesures et la complémentarité de l'AFM et du SEM. Enfin, la dernière partie décrit en détail la plateforme CARMEN et les instruments qui l'équipent (ou qui l'équiperont). D'ores et déjà, la plateforme est notamment prête à répondre en grande partie aux exigences de mesure relatives à la mise en application du décret et de l'arrêté sur la déclaration des substances à l'état nanoparticulaire.

\subsection{Etat de l'art en nanométrologie dimensionnelle}

La mesure de paramètres géométriques (taille et morphologie) d'un nano-objet est incontournable pour l'investigation des nanotechnologies. Ces paramètres se retrouvent au cœur des préoccupations métrologiques des industriels (ex. : microélectronique) et des études sur la toxicité éventuelle des nanoproduits. Il s'agit donc en premier lieu de développer des instruments et des méthodes pour mesurer des dimensions caractéristiques d'objets, des distances et des déplacements dans la gamme allant de $1 \mathrm{~nm}$ à $100 \mathrm{~nm}$ et d'y associer des incertitudes de mesure nanométriques ou subnanométriques.

L'état actuel de l'instrumentation susceptible d'être utilisée dans ce cadre (fig. 1) ainsi que les orientations stratégiques futures des INM montrent que cinq instruments sortent du lot, mais que seuls deux d'entre eux, SEM et AFM, ont véritablement des résolutions inférieures au nanomètre permettent d'effectuer des mesures traçables au SI :

- Les microscopes confocaux ou interférométriques ont des résolutions latérales limitées mais de bonnes résolutions verticales qui les rendent intéressants pour la nanométrologie;

- Les techniques utilisant des palpeurs mécaniques (stylets, profilomètres mécaniques...) restent limitées aux mesures de hauteur des nano-objets. Les mesures latérales sont tronquées à cause du rayon micrométrique des pointes. En outre, la force appliquée sur l'échantillon est très forte et peut l'endommager;

- Les techniques de diffusion de la lumière permettent d'apporter des informations sur la taille des très petites particules, présentes par exemple sur des substrats ou dans des liquides. Par diffusion dynamique de la lumière, il est même possible de mesurer le diamètre équivalent des nanoparticules, mais ces mesures ne sont pas directement traçables au mètre;

- Le SEM est un outil de caractérisation très polyvalent, qui associe une grande vitesse de balayage à une très grande gamme de mesure, en offrant par ailleurs la possibilité de reconstruire les objets en 3D. Il est utilisé sur les lignes de productions industrielles, notamment dans le secteur de la microélectronique, et est largement répandu dans les instituts de métrologie. Les progrès réalisés dans le domaine des sources d'électrons et des composants optiques associés permettent aujourd'hui d'atteindre 
des résolutions latérales inférieures au nanomètre avec les SEM de dernière génération, ce qui les rend compétitifs pour la mesure des nanoparticules. Le mode de fonctionnement du SEM avec détection des électrons secondaires est particulièrement bien adapté à la métrologie dimensionnelle latérale 2D (métrologie de la dimension critique (CD) en microélectronique par exemple) mais le contraste suivant la direction $\mathrm{Z}$ ne donne pas d'information directe sur la topologie de l'échantillon. Une estimation des hauteurs est néanmoins possible à partir des projections 2D et en utilisant une approche stéréoscopique. Cependant, à l'échelle du nanomètre, l'interprétation d'une image SEM requiert des connaissances sur l'interaction électron/matière. La résolution est donc fortement influencée par de nombreux paramètres autres que le simple diamètre du faisceau d'électrons : par exemple l'énergie des électrons, les propriétés électriques du nano-objet ou son état de charge en surface, etc. Dans ce contexte, la simulation et l'utilisation de matériaux de référence joueront un rôle important dans la compréhension de ces phénomènes et la maîtrise des incertitudes de mesures associées. La validité de la mesure doit être établie, en prenant soin par exemple de supprimer les effets de charges. Les TEM (Microscope Electronique à Transmission) ne sont souvent pas retenus par les instituts de métrologie car l'image, fruit de la projection de l'objet sur une surface, rend la mesure précise difficile. De plus, ils sont moins polyvalents que les SEM car ils nécessitent de préparer des substrats transparents aux électrons.

- L'AFM est aussi très répandu dans le monde industriel, dans les laboratoires de recherche ainsi que dans les INM. Il tire son haut pouvoir résolvant de la mise en interaction de l'échantillon à imager avec une fine pointe dont l'extrémité possède un rayon de courbure d'environ $10 \mathrm{~nm}$. Par balayage de la pointe sur l'échantillon, l'AFM est capable de reconstruire une image tridimensionnelle de la surface aussi bien à l'air ambiant que sous vide ou en milieu liquide. Malgré les nombreuses possibilités qu'il offre, son utilisation principale reste l'observation de la topographie d'une surface. Les résolutions alors obtenues sont inférieures au nanomètre verticalement $(Z)$ et dépendent horizontalement (XY) de la taille et de la géométrie de la pointe (élargissement des motifs observés par la pointe).

L'AFM procure donc une résolution latérale inférieure à celle observée par le SEM mais compense en délivrant une mesure suivant $\mathrm{Z}$ quantifiée avec une très faible incertitude là où le SEM ne délivre qu'une information qualitative. Par conséquent, les deux instruments présentent une complémentarité qui permet de mesurer le nano-objet de manière traçable dans les trois dimensions de l'espace [15]. La traçabilité de l'AFM et du SEM est assurée au travers d'une procédure d'étalonnage réalisée grâce à des étalons de transfert (réseaux périodiques $1 \mathrm{D}$ ou 2D). Ces étalons de transfert sont parfaitement connus et étalonnés à l'aide d'AFM dits «métrologiques » (voir $§ 4.2)$.

\subsection{AFM métrologique : vers une traçabilité dimensionnelle à l'échelle du nanomètre}

Dans le SI, l'unité de longueur, le mètre, est définie à partir de l'unité de temps, la seconde, grâce à la fixation de la vitesse de la lumière, $c$, par la Conférence générale des poids et mesures (CGPM) en 1983. La définition de la seconde est fondée sur la durée d'un nombre de périodes défini (9192631 770) de la radiation correspondant à la transition entre deux niveaux hyperfins de l'état fondamental de l'atome de césium 133. Le lien entre les unités de temps et de longueur est établi par la relation caractérisant la propagation des ondes électromagnétiques dans le vide et reliant la longueur d'onde $\lambda$ à la fréquence $v: \lambda=c / v$. La référence de fréquence $v$ peut être fournie par un laser stabilisé sur une transition atomique ou moléculaire.

L'unité de longueur est actuellement mise en pratique grâce aux techniques interférométriques qui permettent de passer d'une longueur d'onde (domaine nanométrique) à une longueur macroscopique à l'échelle du mètre.

À l'échelle du nanomètre, la traçabilité de la mesure peut être schématisée par la pyramide de traçabilité présentée sur la figure 2. Au sommet de la pyramide, se trouvent la définition du mètre et sa conservation au sein des INM grâce à un laser stabilisé en fréquence. Le lien entre la mesure à l'échelle du nanomètre et l'étalon primaire est réalisé grâce à un AFM métrologique (mAFM) qui représente la pierre angulaire de toute la chaîne de traçabilité. Dans le cadre de la traçabilité au SI, il permet de raccorder les mesures dimensionnelles de tous les microscopes électroniques en champ proche et donc de rendre les mesures traçables, comparables et exploitables par l'ensemble de la communauté.

Ce raccordement est effectué grâce à des étalons de transfert qui prennent généralement la forme de réseaux périodiques $1 \mathrm{D}$ ou $2 \mathrm{D}$ dont les propriétés dimensionnelles sont parfaitement connues et étalonnées (voir fig. 2). Les étalons de hauteur de marche (Z) ou de pas de réseau $(\mathrm{XY})$ ont été développés en collaboration avec les industriels et sont pour la plupart déjà disponibles dans le commerce avec un large éventail de dimensions caractéristiques. De nouveaux étalons de transfert sont par ailleurs en cours de développement, notamment dans le domaine de la rugosité de surface à l'échelle nanométrique où les besoins industriels sont importants.

Il existe aujourd'hui dans le monde une vingtaine de mAFM. Ils sont construits (ou en cours de développement) dans les INM. Une première génération de ces instruments de référence a vu le jour à la fin des années 1980, financée par des programmes de recherche nationaux ou internationaux. Ces premiers développements ont permis d'appréhender les différentes problématiques liées à la pratique de la nanométrologie dimensionnelle par AFM. 


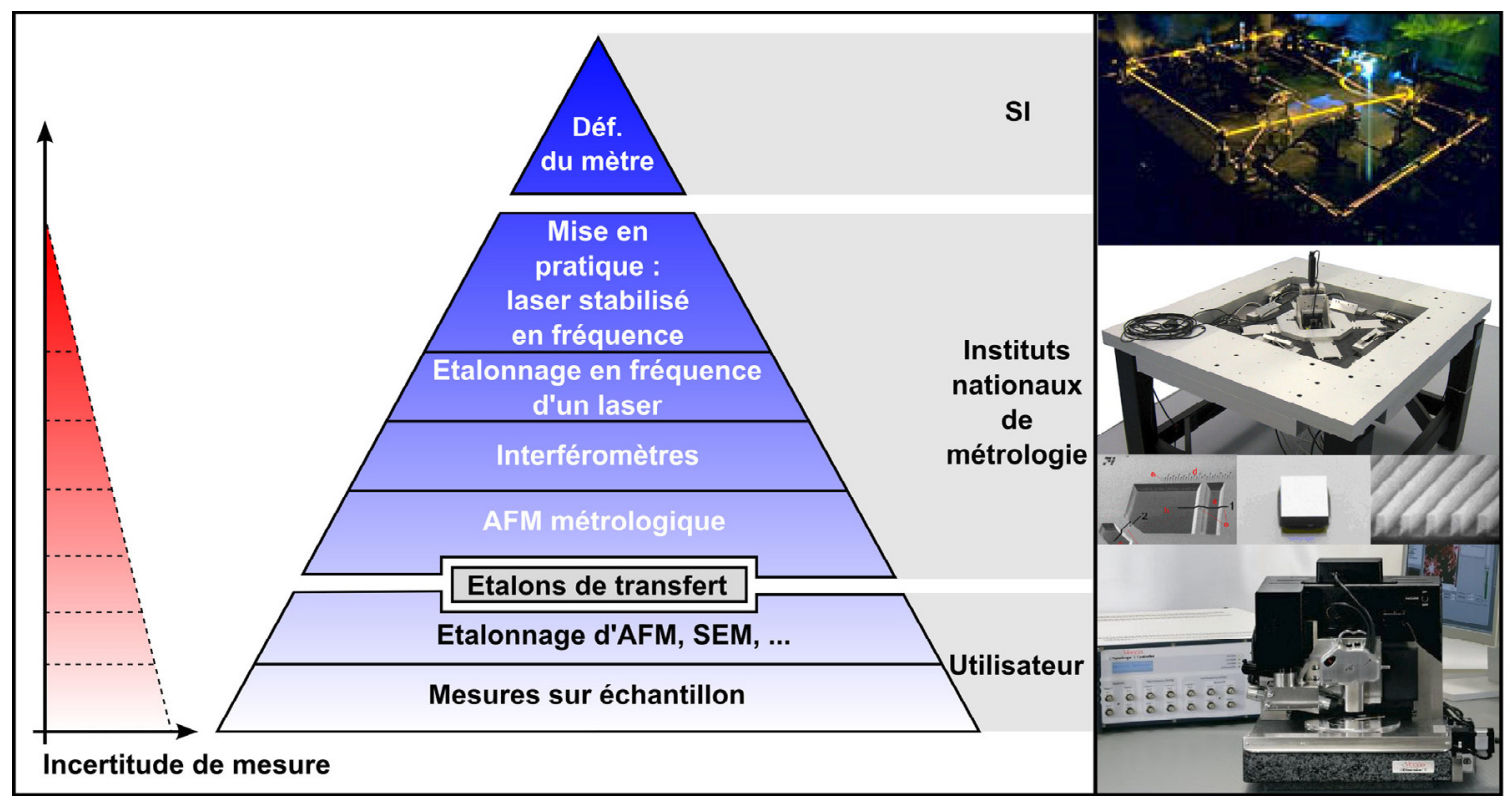

Fig. 2. - Pyramide de traçabilité pour le mètre : à l'échelle du nanomètre, l'AFM métrologique est un moyen de raccordement.

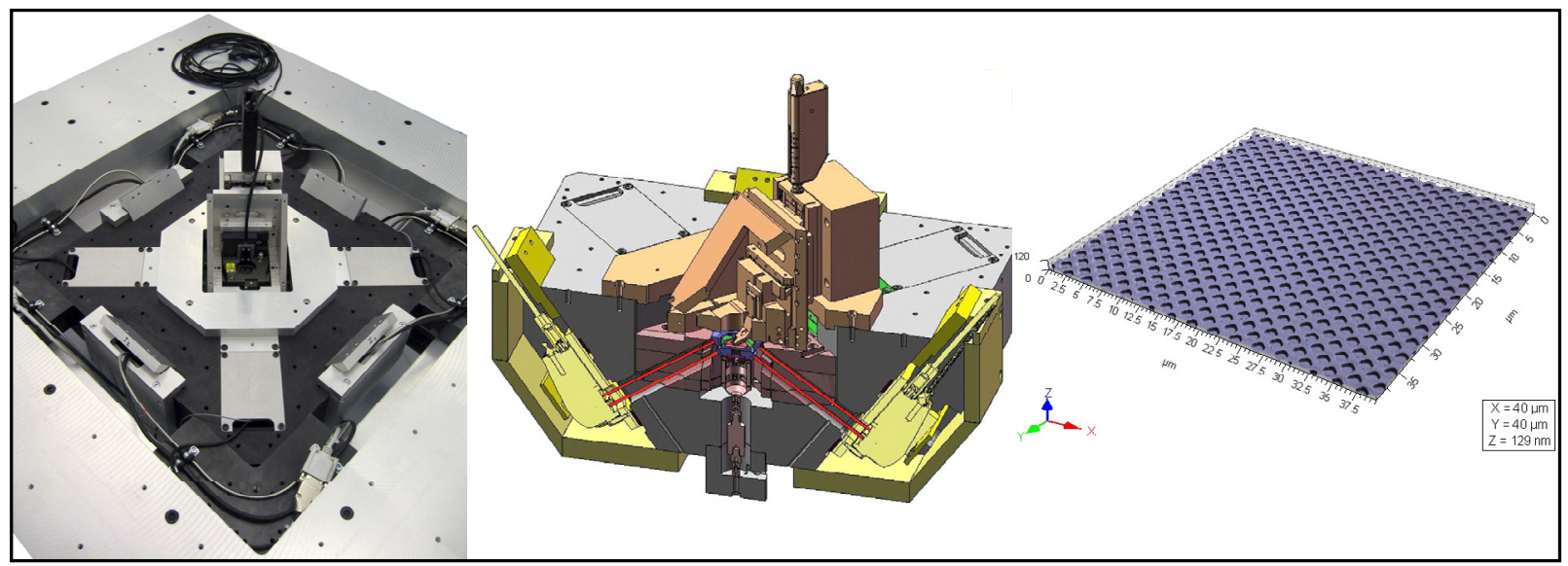

Fig. 3. - À gauche, photographie de l'AFM métrologique du LNE, au centre, vue CAO du mAFM révélant les interféromètres, à droite, image de $40 \mu \mathrm{m} \times 40 \mu \mathrm{m}$ obtenue sur un étalon de transfert (pas de 1,803 $\mu \mathrm{m}$ et hauteur de marche de 41,2 $\mathrm{nm}$ ).

Depuis le début des années 2000, on assiste à l'avènement d'une seconde génération d'instruments qui, riche des expériences acquises sur les premiers mAFMs, propose des solutions différentes, originales, et parfois plus performantes en termes d'incertitudes de mesure notamment. Ces nouveaux équipements permettent d'aborder la compréhension fine des interactions pointe-surface [16].

Le mAFM que développe le LNE depuis 2007 (fig. 3) met en œuvre des interféromètres dont la source laser est étalonnée en longueur d'onde et qui permet de mesurer en temps réel la position relative de la pointe par rapport à l'échantillon. Ainsi, pour chaque point de l'image topographique, les coordonnées XYZ représentent les coordonnées absolues et raccordées au SI. La gamme de déplacements du mAFM est de $60 \mu \mathrm{m} \times 60 \mu \mathrm{m} \times 15 \mu \mathrm{m}$ suivant les axes XYZ. L'incertitude associée à la mesure de la position relative de la pointe par rapport à l'échantillon est de l'ordre du nanomètre. L'instrument a été entièrement développé au LNE afin de maîtriser l'ensemble des choix de conception et minimiser ainsi l'influence des contributions majeures qui pourraient perturber le processus de mesurage et dégrader le résultat (erreur d'Abbe, effets thermiques, contrôle de position par interférométrie dans l'air ambiant, etc.). L'ensemble de ces contributions est regroupé dans un bilan d'incertitude qui caractérise pleinement l'AFM métrologique et qui permettra de calculer l'incertitude de mesure associée à cet instrument. L'étude complète de cet instrument de référence national a fait l'objet d'une thèse de doctorat soutenue en juillet 2010 [17] et au cours de laquelle des premières images ont été obtenues (fig. 3) [18]. Une seconde thèse consacrée au développement d'une tête AFM métrologique dédiée et à la caractérisation métrologique de l'instrument est en cours au laboratoire.

\subsection{Description de la plateforme CARMEN}

La figure 4 donne une description de la plateforme CARMEN. L'ensemble de l'instrumentation est rassemblé dans le laboratoire de nanométrologie du LNE qui comprend $150 \mathrm{~m}^{2}$ de salle blanche, au sein du pôle de 


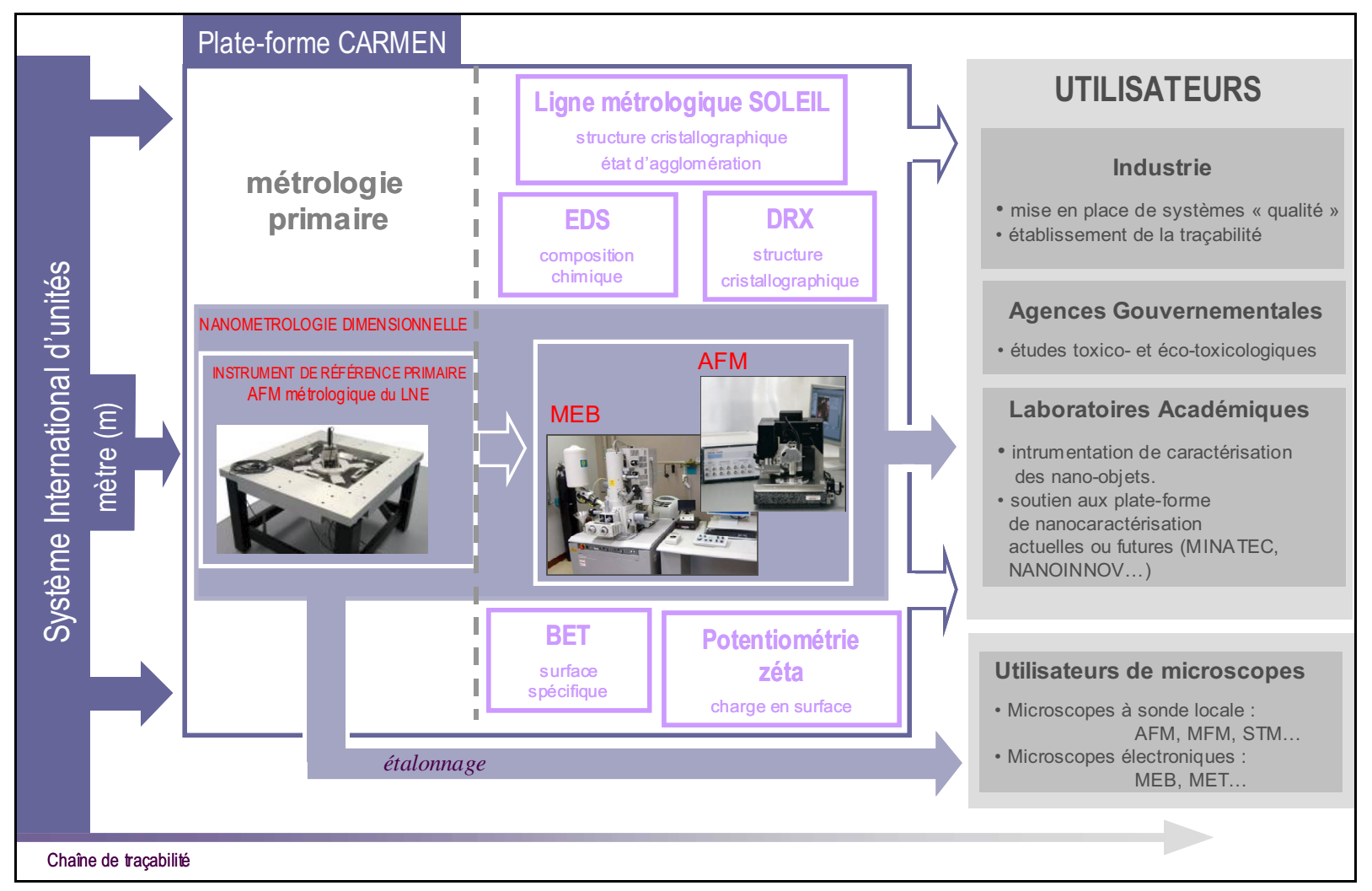

Fig. 4. - Présentation de la plateforme CARMEN du LNE dédiée à la caractérisation métrologique des nanomatériaux.

recherche de métrologie avancée. Ce laboratoire répond aux exigences les plus élevées en termes de :

- stabilité de la température : $(20 \pm 0,1)^{\circ} \mathrm{C}$;

- stabilité en humidité : $(50 \pm 5) \%$.

Les instruments sensibles aux vibrations mécaniques sont installés sur des massifs en béton dissociés des bâtiments.

\subsubsection{La dimension}

Le cœur de la plateforme est dédié à la nanométrologie dimensionnelle. Il est constitué de l'AFM métrologique dont le rôle est de relier les mesures réalisées par un AFM commercial et par un SEM (fig. 5) avec la définition du mètre SI. Le SEM est de type FEG c'està-dire qu'il est muni d'un canon à émission de champ (Field Emission Gun) qui permet d'atteindre des résolutions subnanométriques (fig. 5).

Des travaux sont en cours pour étudier la complémentarité AFM/SEM, pertinente dans le cadre d'une mesure métrologique dans les trois dimensions de l'espace, et plus particulièrement sur la question de la traçabilité des mesures dimensionnelles des nanoparticules via ces deux instruments et le bilan d'incertitudes associées. Les nano-objets utilisés sont des nanoparticules industrielles ou synthétisées dans le cadre de collaborations avec des laboratoires de chimie. Le laboratoire travaille notamment avec une équipe de SCPI/ENSTA-PARISTECH qui a acquis une grande expérience dans le domaine de la synthèse par voie chimique de nanoparticules de taille et de composition contrôlées [19-23].

Un effort particulier est fourni sur le développement de la méthode d'échantillonnage. En effet, dans le cas général, les nanoparticules sont dispersées dans un liquide porteur et la mesure de la taille des particules à partir de l'image du microscope nécessite de déposer la solution colloïdale sur un substrat. Cependant, même dans le cas d'une solution stabilisée, le séchage provoque une forte agglomération des nanoparticules, ce qui empêche souvent de mesurer correctement leur taille et leur distribution en taille. En outre, les solutions commerciales contiennent souvent des produits permettant de stabiliser la solution (par exemple, le citrate est utilisé pour les nanoparticules d'or du NIST), qui conduisent à des dépôts résiduels, souvent observés sur les images AFM et SEM et à l'origine d'une perturbation des mesures. Des stratégies pour disperser et isoler les nanoparticules sur un substrat donné sont donc en cours de développement. Des premières études menées au laboratoire montrent ainsi que le choix du type de substrat est prédominant pour la mesure AFM; la principale caractéristique à rechercher étant notamment une faible rugosité de la surface.

Une méthode d'échantillonnage fondée sur l'utilisation d'une tournette a permis d'obtenir les images présentées sur la figure 6. Cette méthode est constituée de deux phases, la première permet l'étalement de la solution (faible vitesse de rotation) et la seconde permet d'éliminer complètement le liquide porteur (vitesse de 


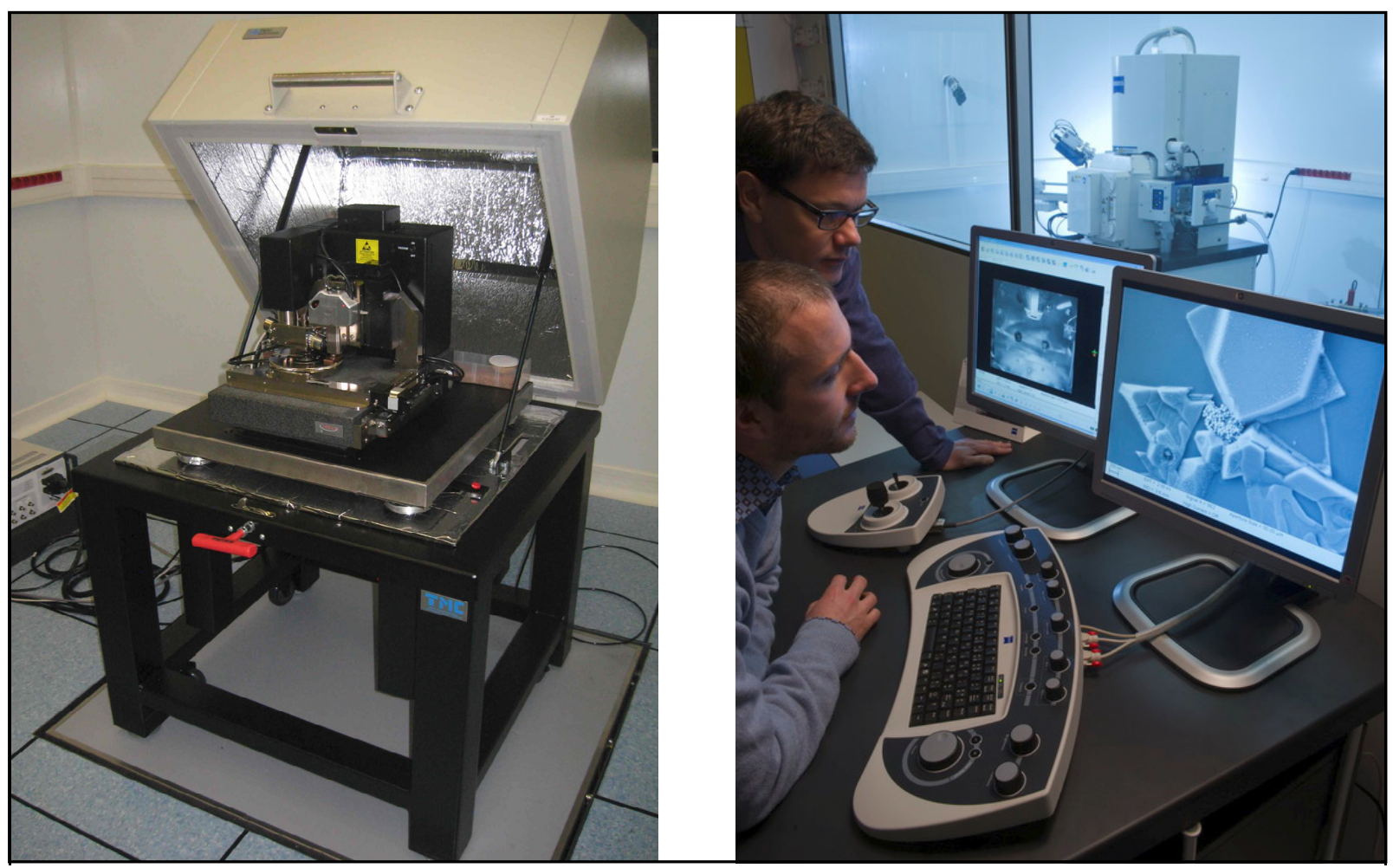

Fig. 5. - L'AFM VEECO et le SEM Ultra-plus ZEISS de la plateforme CARMEN.

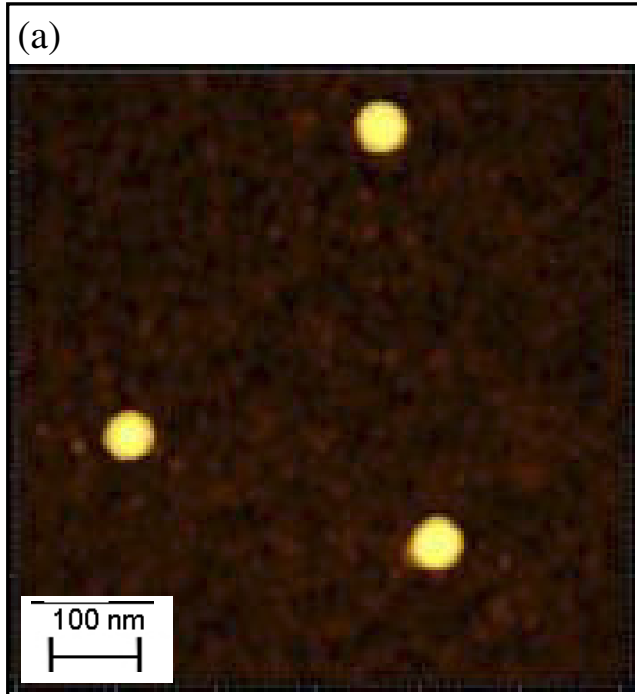

(b)

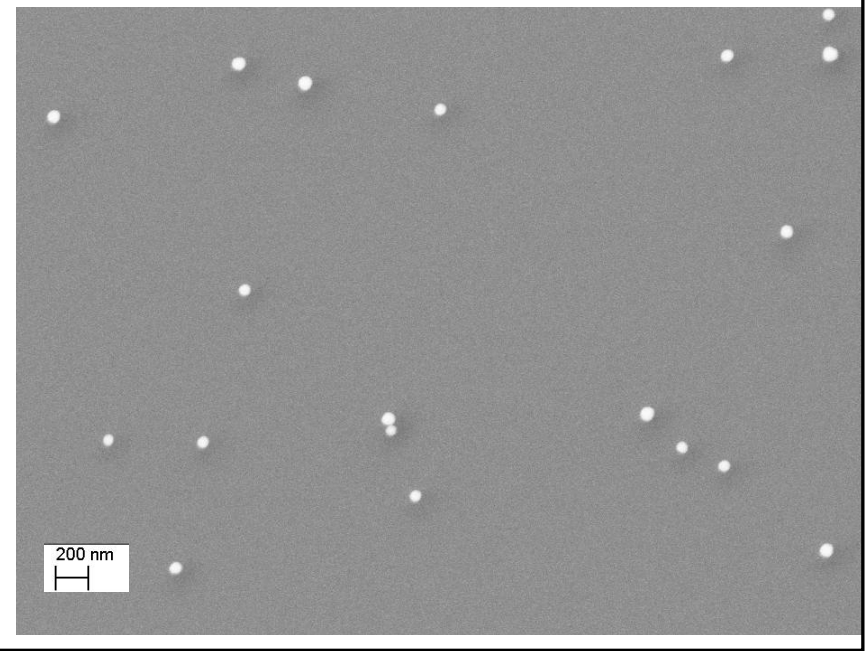

Fig. 6. - Images AFM de nanoparticules d'or commerciales $(60 \mathrm{~nm}$ ) déposées sur un substrat de mica (a) et SEM (b) de nanoparticules synthétisées de $\mathrm{SiO}_{2}(80 \mathrm{~nm})$ déposées sur mica. La méthode de préparation des échantillons permet d'obtenir des nanoparticules correctement dispersées sur le substrat.

rotation élevée). Les clichés ont été obtenus respectivement par AFM (fig. 6a) et SEM (fig. 6b) et ont été effectués sur des solutions colloïdales d'or commerciales de $60 \mathrm{~nm}$ et de $\mathrm{SiO}_{2}$ de $80 \mathrm{~nm}$ déposées sur un substrat de mica.

\subsubsection{La distribution en taille}

La distribution en taille des nanoparticules est aussi un paramètre déterminant comme l'a récemment montré une équipe de l'INM japonais (AIST/NMIJ) dans un article concernant les études toxicologiques et écotoxicologiques [24]. L'étude propose une évaluation de l'incertitude liée à la caractérisation de la dispersion en taille de nanoparticules mesurée en solution et en aérosol.

Le comptage de nanoparticules et la détermination de la taille de chacune d'elles sur un cliché de microscopie est un travail long et fastidieux. Il existe des logiciels de traitement d'image capable de réaliser des comptages automatiques et des déterminations de taille mais ces programmes peuvent induire de nombreuses erreurs. 


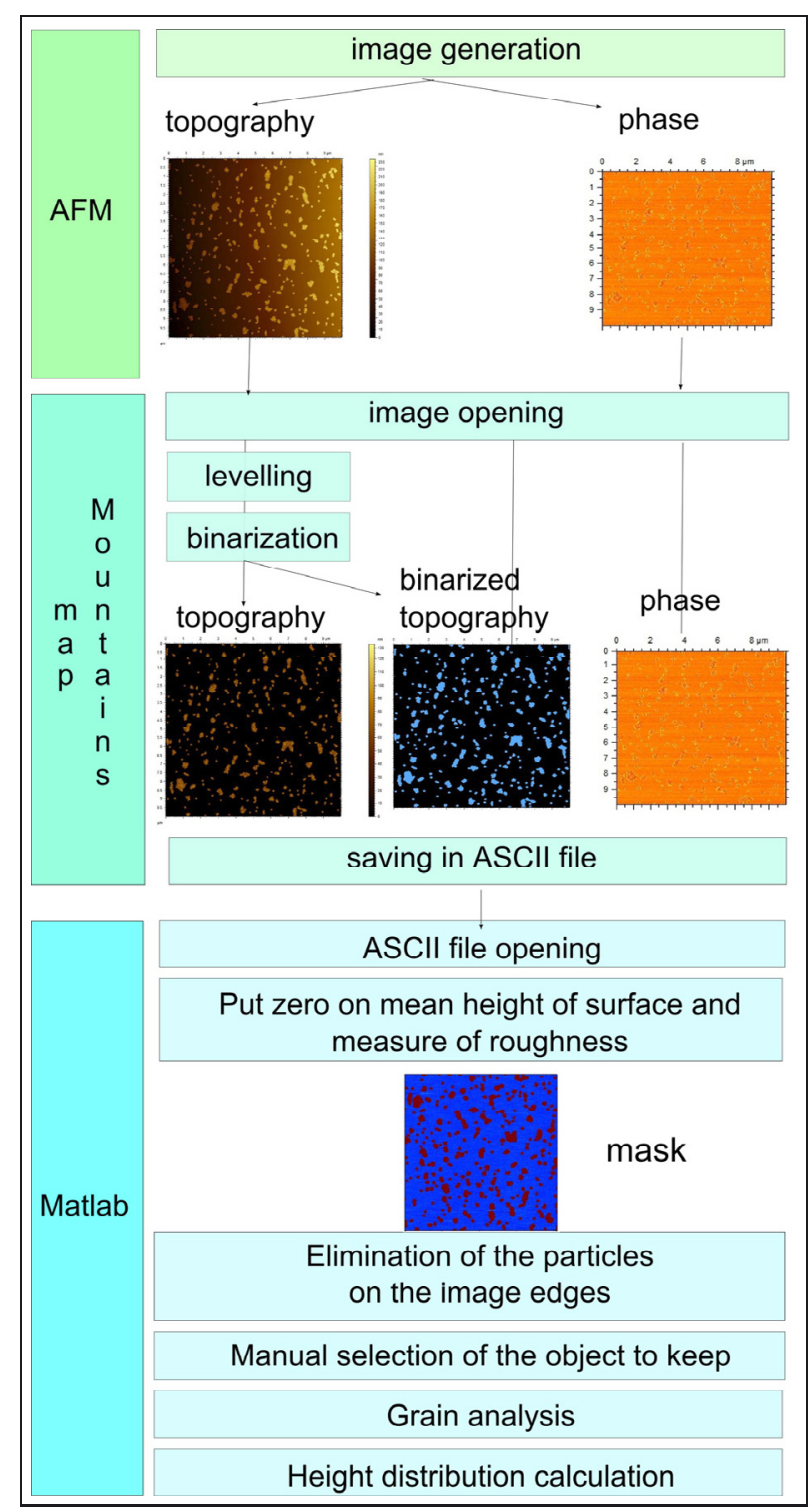

Fig. 7. - Description de la méthode de post-traitement des images.

La plus préjudiciable concerne leur incapacité à faire la différence entre une nanoparticule et un agglomérat de nanoparticules. Chaque agglomérat est donc considéré comme une « grosse » nanoparticule qui entre dans la distribution en taille. Nous avons donc développé une méthode semi-automatique de traitement d'image qui permet de vérifier que chaque objet pris en compte est bien une nanoparticule isolée. Ce programme concerne, pour le moment, la mesure de hauteur de nanoparticules par AFM mais sera bientôt adapté à la mesure SEM. Il est décrit en figure 7 , et comporte deux étapes : binarisation de l'image et comptage des particules.

\subsubsection{Redressement et binarisation de l'image avec Mountains Map (Digital Surf)}

Cette étape consiste à ouvrir une image (topographie et phase) et à redresser l'image de topographie afin de supprimer l'inclinaison due aux dérives thermiques ou au mauvais positionnement de l'échantillon. À l'instar des problèmes liés à la rugosité, un mauvais redressement peut dégrader la qualité de la mesure de hauteur des NP. La fonction line wise correction permet de corriger l'ensemble de l'inclinaison. Cette correction s'effectue ligne par ligne en soustrayant un polynôme d'ordre 1 .

Une fois la surface topographique redressée, l'image est binarisée grâce à la fonction binarization. Un seuil est manuellement défini afin de discriminer correctement le substrat des nano-objets sur la surface : l'ensemble des points au-dessous de ce seuil est attribué au substrat alors que les points au-dessus du seuil entrent dans l'analyse des nano-objets. Cette étape permet d'identifier correctement les NP sur la surface. Evidemment, l'image binarisée perd l'information liée à la hauteur des NP. Les images topographiques redressées, les surfaces binarisées et les images de phase sont conservées dans un fichier ASCII. La figure 8 montre un exemple de trois images (brute, redressée et binarisée) pour des nanoparticules de $\mathrm{SiO}_{2}$ déposées sur du mica selon la méthode décrite précédemment.

\subsubsection{Comptage des nanoparticules et détermination des hauteurs}

La seconde étape a été développée sous Matlab. En pratique, l'image binarisée permet d'identifier et de localiser chaque nanoparticule qui doit être analysée. Un premier filtre est appliqué grâce à la fonction bwselect afin d'exclure automatiquement de la distribution les nanoparticules non complètement imagées qui se trouvent sur les bords de l'image. Cette image corrigée est alors analysée en utilisant la fonction $b$ wlabel pour détecter et numéroter chaque NP isolée. Finalement la fonction regionprops permet une mesure directe du centroïde de la NP et l'isole dans une boîte de délimitation comme le montre la figure 9 .

En parallèle, la détermination de la hauteur de chaque objet est réalisée à partir de l'image générée par Mountains Map (image redressée). La qualité de la mesure dépend du choix correct du point de référence sur l'image. Les mesures de hauteurs par AFM doivent être effectuées par rapport à la ligne moyenne de la rugosité. Afin de déterminer le plus correctement possible ce plan moyen, un masque est appliqué sur la topographie (image redressée) pour exclure les NP et leur environnement proche et ne garder que la topographie du substrat. Ce masque est réalisé grâce à l'image binarisée. La valeur moyenne des points calculés à partir de l'image redressée (après soustraction du masque) correspond au plan moyen de la rugosité. Ce plan de référence représente le niveau zéro de l'image topographique et toutes les mesures de hauteurs de nanoparticules seront réalisées par rapport à ce plan. Le paramètre $R_{\mathrm{q}}$ de la rugosité peut être ainsi déterminé.

À partir de l'image redressée et de notre plan de référence correctement déterminé, la mesure de la hauteur des nanoparticules peut être effectuée et correspond au maximum local identifié dans la boîte de délimitation de 


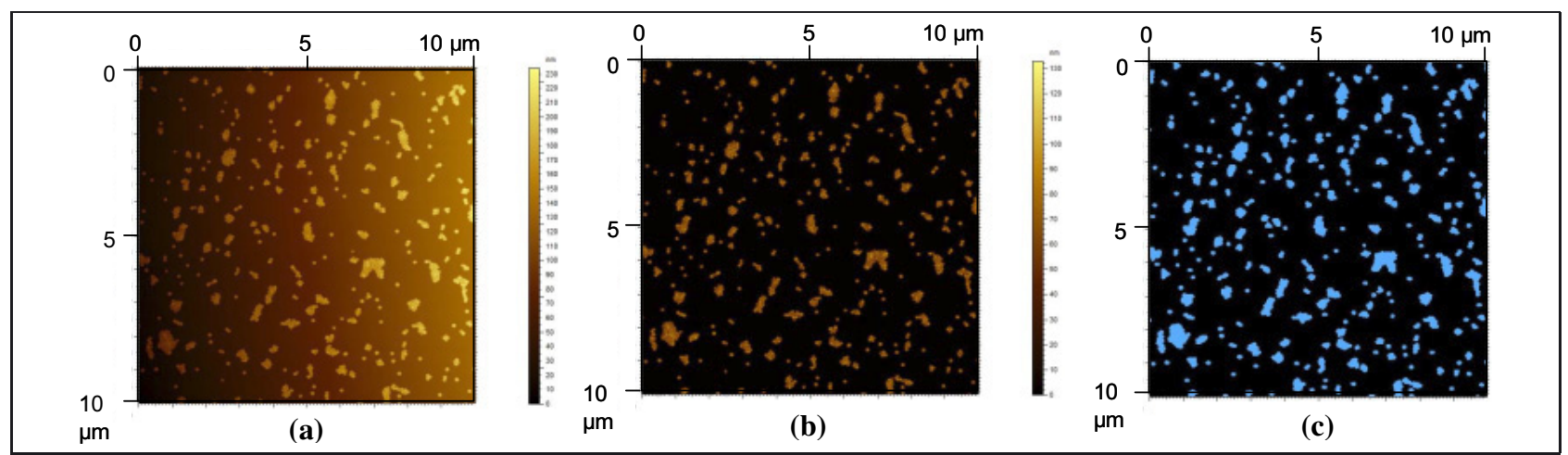

Fig. 8. - Images de nanoparticules de $\mathrm{SiO}_{2}$ déposées sur du mica : (a) image brute AFM, (b) image redressée et (c) image obtenue après le processus de binéarisation.

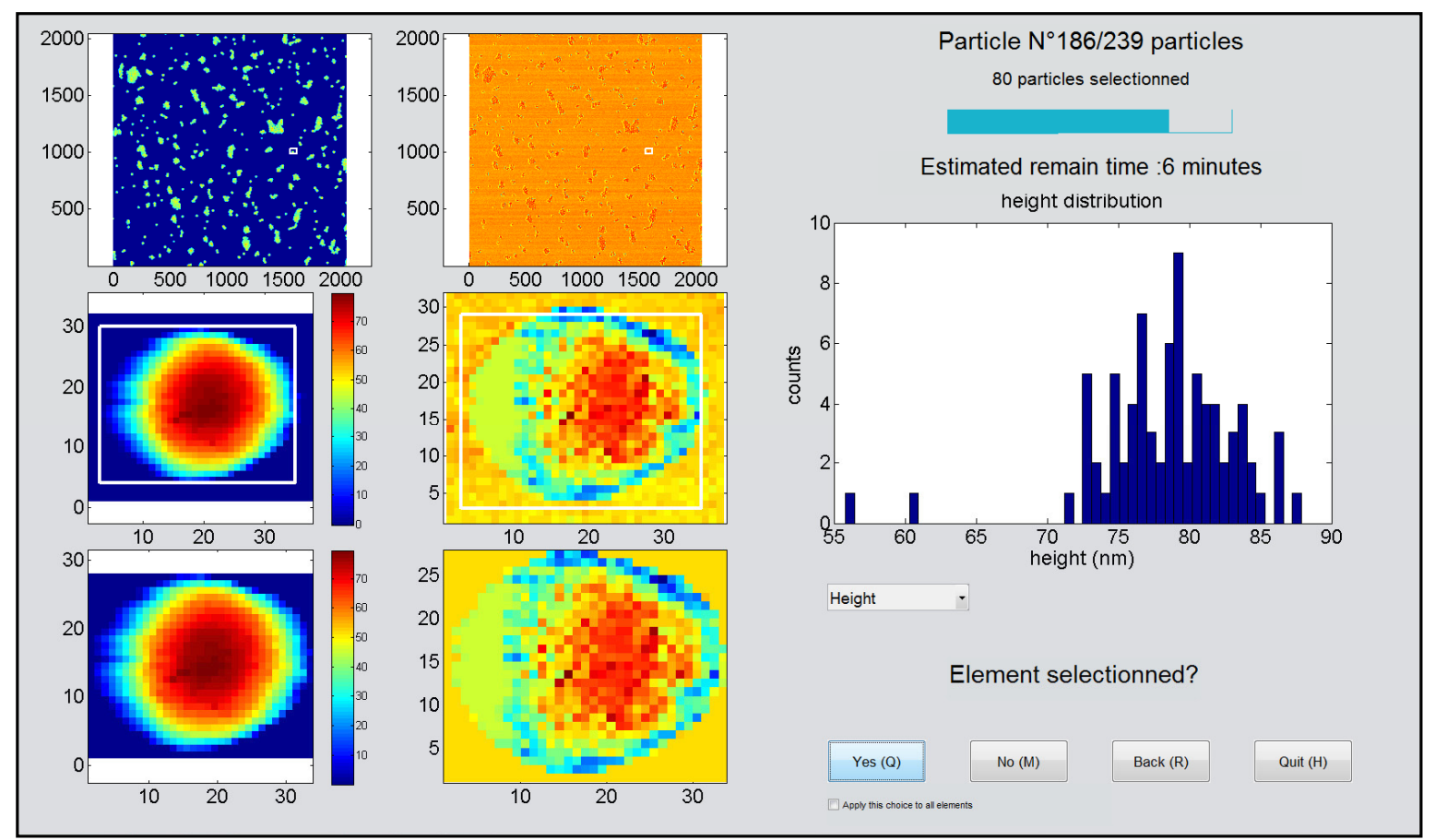

Fig. 9. - Copie d'écran de l'interface du programme développé sous Matlab. Sur la partie gauche, en haut, l'image des NP en topographie et en dessous une particule isolée, identifiée par l'image binéarisée et agrandie dans la boîte de délimitation. Au milieu, même traitement pour les images en phase. À droite, l'histogramme en cours de construction. Les boutons «yes » et «no» permettent de prendre en compte ou non l'objet si celui-ci est un agglomérat ou une NP isolée.

l'objet (fig. 9). L'interface comporte des boutons « yes» or «no» qui permettent d'accepter ou non la prise en compte de l'objet analysé. L'opérateur peut alors éliminer ainsi tous les agglomérats de NP qui sont facilement identifiables grâce à la boîte de délimitation de l'image du nano-objet en topographie et phase. L'image en phase est souvent plus contrastée que l'image en topographie et permet de mieux identifier les limites de l'objet; elle apporte ainsi des informations supplémentaires. Cette méthode semi-automatique évite les erreurs de comptage dans la distribution en taille et dans le calcul de la valeur moyenne des diamètres de NP. Une fois la NP prise en compte, elle est ajoutée à l'histogramme qui se construit en temps réel.

Le caractère rapide de ce traitement semiautomatique permet de traiter plus d'images et donc de prendre en compte dans la statistique plus de particules qu'un traitement entièrement manuel. Grâce à cette technique, il est aisé d'atteindre les 100 nanoparticules nécessaires afin que la distribution en taille déterminée soit représentative de l'ensemble de la population [25] de la solution colloïdale de départ. Ce traitement sera par la suite adapté aux images SEM afin de mieux étudier la complémentarité AFM/SEM.

Une population de nanoparticules de $\mathrm{SiO}_{2}$ déposées sur mica a ainsi été analysée par AFM en utilisant le programme développé. Les hauteurs de 389 nanoparticules ont été mesurées à partir de quatre images AFM de $5 \mu \mathrm{m} \times 5 \mu \mathrm{m}(2048 \times 2048$ pixels $)$. L'histogramme à la fin du processus de comptage est donné en figure 10 . Le diamètre moyen $\left(D_{\mathrm{m}}=79,05 \mathrm{~nm}\right)$ et l'écart type $(\sigma=5,67 \mathrm{~nm})$ de la population ont été calculés et les 


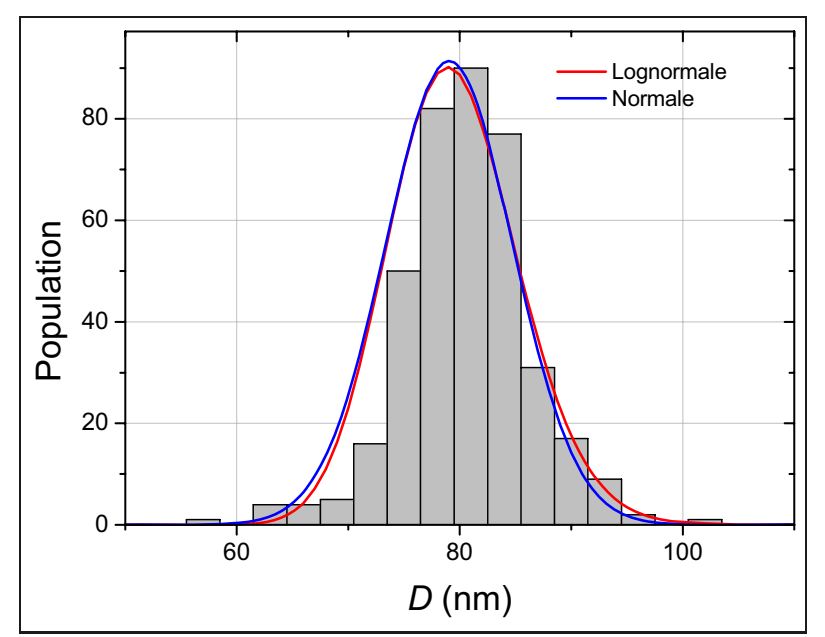

Fig. 10. - Histogramme de distribution en taille (hauteur) de nanoparticules de $\mathrm{SiO}_{2}$ sphérique. Les lois «log-normale » et «normale » ont été ajustées.

valeurs ont été utilisées pour tracer les lois log-normale et normale superposées sur le graphe. Des tests d'adéquation ont été effectués mais n'ont pas permis d'éliminer une loi statistique par rapport à l'autre.

\subsubsection{La forme et l'état d'agrégation/agglomération}

La forme des nano-objets étudiés est un élément déterminant qui peut se révéler important notamment dans le cas des études toxicologiques. Par exemple, des études ont montré que le $\mathrm{TiO}_{2}$ sous forme fibreuse est plus dangereux que sous forme sphérique [26]. De plus, les informations à déterminer peuvent être la forme du nano-objet primaire (nanoparticule sphérique, bâtonnet, nanotube, fibres par exemple) ou la forme d'un objet qui est le résultat d'un processus d'agrégation ou d'agglomération de plusieurs nano-objets primaires.

L'agglomérat est une association de particules liées par des forces faibles (Van der Waals, électrostatiques ou tension superficielle), chaque particule étant tangente à ses voisines. La morphologie des agglomérats n'est pas une propriété du nanomatériau mais le fruit d'un état temporaire d'un équilibre dynamique entre les effets de dispersion et d'agglomération dans une suspension ou un aérosol.

L'agrégat quant à lui est une nanoparticule hétérogène dans laquelle les différents constituants sont reliés par des liaisons fortes (covalentes). Il peut être le fruit d'une fusion entre plusieurs nanoparticules primaires, le régime d'agrégation conditionnant la forme finale de l'agrégat (compacité, dimensions, etc.) et son interaction avec le milieu dans lequel il se trouve (pénétration du système respiratoire et des cellules).

$\mathrm{Au}$ niveau international, les mesurandes définissant les paramètres de forme, d'agrégation et d'agglomération sont encore à établir ainsi que les protocoles de mesure. Un des paramètres pertinents pour étudier la morphologie d'un agrégat ou d'un agglomérat de nano-objets est la dimension fractale, définie par le mathématicien français Benoît Mandelbrot et qui traduit le rapport entre, par exemple, son volume et son rayon de giration ou sa surface et son périmètre. Le cœur dimensionnel de la plateforme CARMEN est totalement adapté à la mesure de tout paramètre définissant la forme d'un nano-objet ou d'un agrégat/agglomérat de nano-objet. De plus, la mise en place d'une traçabilité au niveau national des mesures AFM et SEM, actuellement en cours de développement, peut permettre de renforcer le niveau de confiance des calculs de la dimension fractale des nano-objets.

\subsubsection{La composition chimique}

Dans le contexte des études toxicologiques et écotoxicologiques, la nature métallique ou la présence d'atomes de métaux de transition peuvent être des facteurs aggravants de la dangerosité des nano-objets. En effet, les métaux de transition interviennent dans des réactions induisant des formes réactives de l'oxygène. Si la nature chimique des nanomatériaux ne peut être considérée comme un facteur exclusif des études toxicologiques, il est tout de même nécessaire de la déterminer avec précision.

Comme dans la plupart des laboratoires de nanocaractérisation, la composition chimique est obtenue grâce à l'imagerie élémentaire effectuée par spectrométrie de rayon $\mathrm{X}$ associée au SEM de la plateforme CARMEN. La présence d'impuretés à la surface des nanoparticules peut également expliquer une toxicité éventuelle, cependant une instrumentation complémentaire à celle de la plateforme CARMEN est nécessaire (spectroscopie infrarouge, spectrométrie de masse d'ions secondaires [27]...).

\subsubsection{La structure cristallographique}

La nature des phases cristallines d'une population de nanoparticules peut, dans certains cas, jouer un rôle sur la toxicité éventuelle de ces dernières. Par exemple, les nanoparticules manufacturées de dioxyde de titane $\left(\mathrm{TiO}_{2}\right)$ existent dans trois structures cristallines : rutile, anatase et brookite. Selon la structure, les études toxicologiques donnent des résultats très différents [28].

La diffraction des rayons $\mathrm{X}$ (DRX) permet de déterminer les différentes phases cristallographiques présentes dans un nano-objet en comparant la position des pics du diffractogramme avec les fiches internationales de diffraction de l'ICDD (International Centre for diffraction Data). Le diffractogramme permet aussi de déterminer les paramètres de maille d'un réseau cristallin, les positions atomiques (méthode de Rietveld) et de calculer un diamètre équivalent, $D_{\mathrm{DRX}}$, des nano-objets grâce à l'élargissement des pics (méthode de Scherrer). Cependant, d'autres facteurs peuvent élargir ses pics et venir biaiser la mesure de $D_{\mathrm{DRX}}$ comme par exemple des phases d'amorphisation en surface d'une nanoparticule. Ces désordres locaux augmentent d'autant plus que les dimensions caractéristiques du nano-objet diminuent. Des études sont en cours dans différents laboratoires autour de la compréhension des diffractogrammes et des 
différents paramètres caractéristiques que l'on peut en tirer. Les INM européens cherchent à développer des méthodes de référence, des outils de simulation et à élaborer des matériaux de référence [15].

Un diffractomètre à rayon $\mathrm{X}$ sera installé dans les salles blanches du laboratoire de nanométrologie. Il s'agit d'un diffractomètre X'pert Pro MPD qui possède un goniomètre spécifique pour des mesures aux grands angles. Il est particulièrement bien adapté pour la caractérisation de poudres.

Toutefois, les informations déduites des mesures en diffraction $X$ peuvent se révéler insuffisantes pour décrire la structure intime du nano-objet. Des mesures complémentaires déterminantes sont alors obtenues grâce à des mesures d'absorption $\mathrm{X}$ ou de diffusion $\mathrm{X}$ en utilisant les sources de rayonnement synchrotron. Le LNE peut accéder à certaines lignes de lumière du Centre de rayonnement synchrotron SOLEIL à Saclay. Cela permettrait de mener des investigations supplémentaires sur la structure des nano-objets.

\subsubsection{La charge en surface}

Des études sur des nanoparticules de latex chargées positivement montrent qu'elles sont piégées dans le mucus épithélial chargé négativement, alors que les nanoparticules électronégatives le traversent et peuvent être captées par les entérocytes (cellules de l'épithélium intestinal, au sein de la muqueuse intestinale) [29]. L'état de charge en surface des nano-objets peut donc être un facteur pertinent des études toxicologiques. Il peut être déterminé par potentiométrie zéta. Le potentiel zéta correspond à la charge acquise par une particule solide grâce aux ions qui l'entourent dans une solution colloïdale. La mesure de ce potentiel est souvent la clé pour comprendre les processus d'aggrégation et de dispersion de particules primaires. Les potentiomètres mesurent la mobilité électrophorétique de la suspension chargée dans un champ électrique et permettent de déduire la charge portée par chaque particule, ainsi qu'une taille hydrodynamique.

Le zétamètre de la plateforme CARMEN a été financé par le Domaine d'Intérêt Majeur de la région Ile de France (DIM-IDF) «Des atomes froids aux nanosciences ». L'appareil est équipé d'un système DLS (Dynamic Light Scattering) qui permet la mesure de profil de distribution en taille de suspension collö̈dale de nanoparticules. Le laboratoire sera ainsi en mesure de déterminer la charge d'une population de nanoparticules et les distributions en taille obtenues par DLS pourront alors être comparées à celles obtenues avec le cœur dimensionnel (AFM, SEM). De plus, le zétamètre est un instrument incontournable pour améliorer la méthode de préparation d'échantillons destinés aux études métrologiques dimensionnelles des nano-objets mesurés en AFM ou SEM.

\subsubsection{La surface spécifique}

Lorsque la taille des nanoparticules diminue, le rapport entre la surface et le volume peut dépasser $50 \%$
(D $<4 \mathrm{~nm}$ ). Ceci signifie qu'en dessous d'un certain seuil, plus de la moitié des atomes constitutifs du nanoobjet sont à la surface. Ces atomes se trouvent dans une position inconfortable car il leur manque des voisins. Il apparait ainsi des liaisons pendantes qui expliquent la forte réactivité chimique des nano-particules. La méthode BET (Brunauer, Emmet et Teller, 1938) permet de déterminer la surface totale géométrique (surface spécifique) du nano-objet grâce à l'adsorption de molécules de gaz. Elle constitue la référence pour les études toxicologiques ainsi que pour la caractérisation de la surface spécifique des poudres (chimie catalytique, par exemple). Cette méthode consiste à mettre en contact le matériau à mesurer avec un gaz inerte (azote, par exemple), puis à construire des isothermes expérimentales de physisorption traduisant la quantité de molécules adsorbées à la surface du solide en fonction de leur quantité disponible, exprimée en termes de pression. Les problèmes liés à la mesure de la surface par BET commencent à peine à être étudiés dans les INM européens [15]. Cette méthode de mesure a été identifiée par l'arrêté gouvernemental de déclaration des substances à l'état nanoparticulaire pour la mesure de la surface spécifique.

\section{Conclusion}

Les nanosciences sont pluridisciplinaires et font intervenir l'ensemble des domaines de la recherche scientifique (physique, chimie, biologie). Cette particularité rend le «nanomonde» à la fois riche et complexe. Dans le domaine de la caractérisation, l'investigation des propriétés des nano-objets demande de multiples instruments, parfois très coûteux, un environnement extrêmement bien contrôlé, et une expertise importante.

Dans ce contexte, en soutien à l'industrie et au secteur académique, la plateforme CARMEN développée par le LNE apportera des moyens métrologiques d'excellence fonctionnant en environnement contrôlé et fournissant les meilleures incertitudes de mesure. Elle viendra en aide aux industriels (notamment aux PME) qui ne possèdent pas les instruments nécessaires et contribuera aux études de toxicologie des nano-produits.

Pour répondre à ces objectifs, la plateforme CARMEN intègre un AFM commercial et un SEM qui, à l'aide de l'AFM métrologique réaliseront des mesures traçables à la définition du mètre SI. À terme, l'ensemble des instruments de la plateforme permettra de mesurer les paramètres identifiés comme pertinents (la dimension, la dispersion en taille, la composition chimique, la structure cristallographique, la morphologie, l'état d'agrégation/agglomération, la surface spécifique, la charge en surface et la chimie de surface) pour la caractérisation des nano-objets.

Sur l'ensemble des paramètres mesurés par la plateforme CARMEN, le travail consistera donc à établir les différentes chaînes de traçabilité, à définir des protocoles de mesure, des méthodes d'échantillonnage afin d'être capable d'associer une incertitude à chaque mesure et de permettre d'offrir aux utilisateurs de la plateforme un 
niveau de confiance optimum. De plus, il s'agira de venir en soutien des études sur la normalisation et de faire entendre la voix de la France au niveau international.

Enfin, le LNE et le C'Nano ont créé en 2011 un club de nanométrologie qui compte à ce jour environ 220 membres dont un tiers d'industriels (www. club-nanometrologie.fr). Ce club a pour objectif principal de créer un réseau national d'utilisateurs d'instruments dédiés à la mesure de nano-objets. Dans le cadre des activités du LNE, les informations collectées au sein de ce club permettront de renforcer la stratégie de développement de la plateforme CARMEN.

\section{Références}

[1] «Les nanomatériaux - Effets sur la santé de l'homme et sur l'environnement », Rapport d'expertise collective, AFSSET, juillet 2006.

[2] «Evaluation des risques liés aux nanomatériaux pour la population générale et pour l'environnement », Rapport d'expertise collective, AFFSET, mars 2010.

[3] "Scientific opinion: the potential risks arising from nanoscience and nanotechnologies on food and feed safety", The EFSA Journal, 959, 2009, 1-39.

[4] «Evaluation biologique des dispositifs médicaux contenant des nanomatériaux », Rapport NanoDM, AFSSAPS, février 2011.

[5] «Nanomatériaux : une approche de la sécurité au cas par cas pour des technologies innovantes », Communiqué de presse, Commission Européenne, 3 octobre 2012.

[6] ISO, TS 27687:2008, « Nanotechnologies - Terminologie et définitions relatives aux nano-objets - Nanoparticule, nanofibre et nanofeuillet ».

[7] GoodNanoGuide : «What are nanomaterials?». http://goodnanoguide.org/tiki-index.php? page $=$ What + are + nanomaterials .

[8] Recommandation de la commission du 18 octobre 2011 relative à la définition des nanomatériaux, L 275/38, JOUE, 20 octobre 2011

[9] "Nanoscience and nanotechnologies: opportunities and uncertainties", Rapport de la "Royal Society » et de la «Royal Academy of Engineering, www.nanotec.org.uk/ finalReport.htm.

[10] CARNEIRo K., Nanometrology, 4, 2002, 7.

[11] PURVIS G., "Nanotechnology, standards and tools benefit smart materials", Smart Materials Bulletin, 1, 2002, 9-12.

Article reçu le 22 mai 2012, version révisée reçue le $1^{\text {er }}$ février 2013.
[12] «Intel lance les premiers processeurs gravés en $22 \mathrm{~nm}$ », Usine Nouvelle, avril 2012.

[13] Rapport de l'ITRS sur la métrologie accessible sur le site : http://www.itrs.net/Links/2009ITRS/Home2009.htm.

[14] Résolution du parlement européen su 24 avril 2009 sur les aspects réglementaires des nanomatériaux (2008/22085INI)), 2010/C 184 E/18, juillet 2010.

[15] "Nanometrology Discussion papers 2010", Co-Nanomet Workgroup, 2010

[16] YACOOT A. et al., "Aspects of scanning force microscope probes and their effects on dimensional measurement", Journal of Physics D: Applied Physics, 41, 2008, 1-46.

[17] Poyet B., «Conception d'un microscope à force atomique métrologique », Thèse de doctorat en sciences, 8 juillet 2010, Université de Versailles Saint-Quentin en Yvelines.

[18] Ducourtieux S. et Poyet B., "Development of a metrological atomic force microscope with minimized Abbe error and differential interferometer real-time position control", Measurement Science and Technology, 22, 9, 2011, 94010 .

[19] Palard M. et coll., Materials Chemistry and Physics, 120, 2010, 79-88.

[20] Balencie J. et coll., Materials Chemistry and Physics, 112, 2008, 546-550.

[21] Coudun C. et coll., Catalysis Today, 124, 2007, 49-54.

[22] Coudun C. et Hochepied J.-F., J. Phys. Chem. B, 109, 2005, 6069-6074.

[23] Almeida DE Oliveira A.P. et coll., Chem. Mater. (2003), Vol. 15(16) ; pp. 3202-3207.

[24] Eharal K. et SaKurai H., "Metrology of airbone and liqui-borne nanoparticles: current status and future needs », Metrologia, 47, 2010, S83-90.

[25] SoNG N.W. et al., "Uncertianty estimation of nanoparticle size distribution from a finite number data obtained by microscopic analysis", Metrologia, 46, 2009, 480-488.

[26] R.F. HAMiLton Jr et coll., « Particle length-dependent titanium dioxide nanomaterials toxicity and bioactivity », Particle and fibre Toxicology, vol. 6, article 35, 2009.

[27] GougET B. et CARRIÈRE M., « Nanomatériaux et risques humains au poste de travail », Techniques de l'ingénieur, SL 6 410-1.

[28] IAVICOLI et coll., "Toxicological effects of titanium dioxide nanoparticles: a review of in vitro mammalian studies", Eur. Rev. Med. Pharmacol. Sci., 15, 2011, 481.

[29] FröHLich E. et RoblegG E., "Models for oral uptake of nanoparticles in consumer products", Toxicology, 291, $2012,10$. 